\title{
The Magdalena Ridge Observatory interferometer: 2014 status update
}

\author{
M. J. Creech-Eakman ${ }^{\mathrm{a}}$, V. Romero ${ }^{\mathrm{a}}$, I. Payne ${ }^{\mathrm{a}}$, C.A. Haniff ${ }^{\mathrm{b}}$, D.F. Buscher ${ }^{\mathrm{b}}$, C. Dahl ${ }^{\mathrm{a}}$, A. Farris ${ }^{\mathrm{a}}$, M. \\ Fisher $^{\mathrm{b}}$, C. Jurgenson ${ }^{\mathrm{c}}$, D. Klinglesmith ${ }^{\mathrm{a}}$, T. McCracken ${ }^{\mathrm{c}}$, M. Napolitano ${ }^{\mathrm{a}}$, A. Olivares ${ }^{\mathrm{a}}$, J. Riker ${ }^{\mathrm{a}}$, S. \\ Rochelle $^{\mathrm{a}}$, C. Salcido ${ }^{\mathrm{a}}$, F. Santoro ${ }^{\mathrm{d}}$, L. Schmidt ${ }^{\mathrm{a}}$, R. Selina ${ }^{\mathrm{e}}$, E. B. Seneta ${ }^{\mathrm{b}}$, A. Shtromberg ${ }^{\mathrm{a}}$, X. Sun ${ }^{\mathrm{b}}$, \\ D.M.A. Wilson ${ }^{\mathrm{b}}$, J.S. Young ${ }^{\mathrm{b}}$ \\ a - Magdalena Ridge Observatory, New Mexico Institute of Mining and Technology, 801 Leroy Place, Socorro, NM, \\ 87801. \\ b - Cavendish Laboratory, University of Cambridge, JJ Thomson Avenue, Cambridge, UK, CB30HE. \\ c - Yale University, Astronomy Department, 260 Whitney Avenue, New Haven, CT 06511. \\ d-GMT, GMTO Corporation, 251 S. Lake Ave, Suite 300, Pasadena, CA, 91101 \\ e - NRAO, AOC, 1003 Lopezville Road, Socorro, NM, 87801.
}

\begin{abstract}
The Magdalena Ridge Observatory Interferometer has been designed to be a $10 \times 1.4 \mathrm{~m}$ aperture long-baseline optical/near-infrared interferometer in an equilateral "Y" configuration, and is being deployed west of Socorro, NM on the Magdalena Ridge. Unfortunately, first light for the facility has been delayed due to the current difficult funding regime, but during the past two years we have made substantial progress on many of the key subsystems for the array. The design of all these subsystems is largely complete, and laboratory assembly and testing, and the installation and site acceptance testing of key components on the Ridge are now underway. This paper serves as an overview and update on the facility's present status and changes since 2012, and the plans for future activities and eventual operations of the facilities.
\end{abstract}

Keywords: Optical interferometer, telescope facilities, high-resolution imaging, fringe tracking, beam combiners, delay lines, alignment systems

\section{MAGDALENA RIDGE OBSERVATORY INTERFEROMETER OVERVIEW}

The Magdalena Ridge Observatory (MRO) is a Federal, State and institutionally funded facility being built and managed by New Mexico Institute of Mining and Technology (NMT). NMT also serves as host for the observatory offices at its campus in Socorro, NM. The observatory consists of two major facilities: a fast-tracking $2.4 \mathrm{~m}$ telescope and a multielement optical/infrared interferometric array (Figure 1). The interferometer is being designed and built in collaboration with partners at the University of Cambridge, UK. Previous employees of MROI continue to participate in subsystem work as well. Many details of both the general design of the array, its specific subsystems and its scientific foci have been discussed in the last several SPIE topical meetings on optical interferometry, and so this paper will serve as a status update on the facilities since the SPIE 2012 paper (Creech-Eakman et al. 2012). The MROI conceptual design was recently published (Buscher et al. 2013) in order to capture the design decisions behind the detailed hardware choices that have gone into building the MROI. In the following sections below we discuss the project's progress over the past two years and present our current plans for the future facility deployment and its operational phase, which are heavily dependent on the near-term funding profile for the facility. 


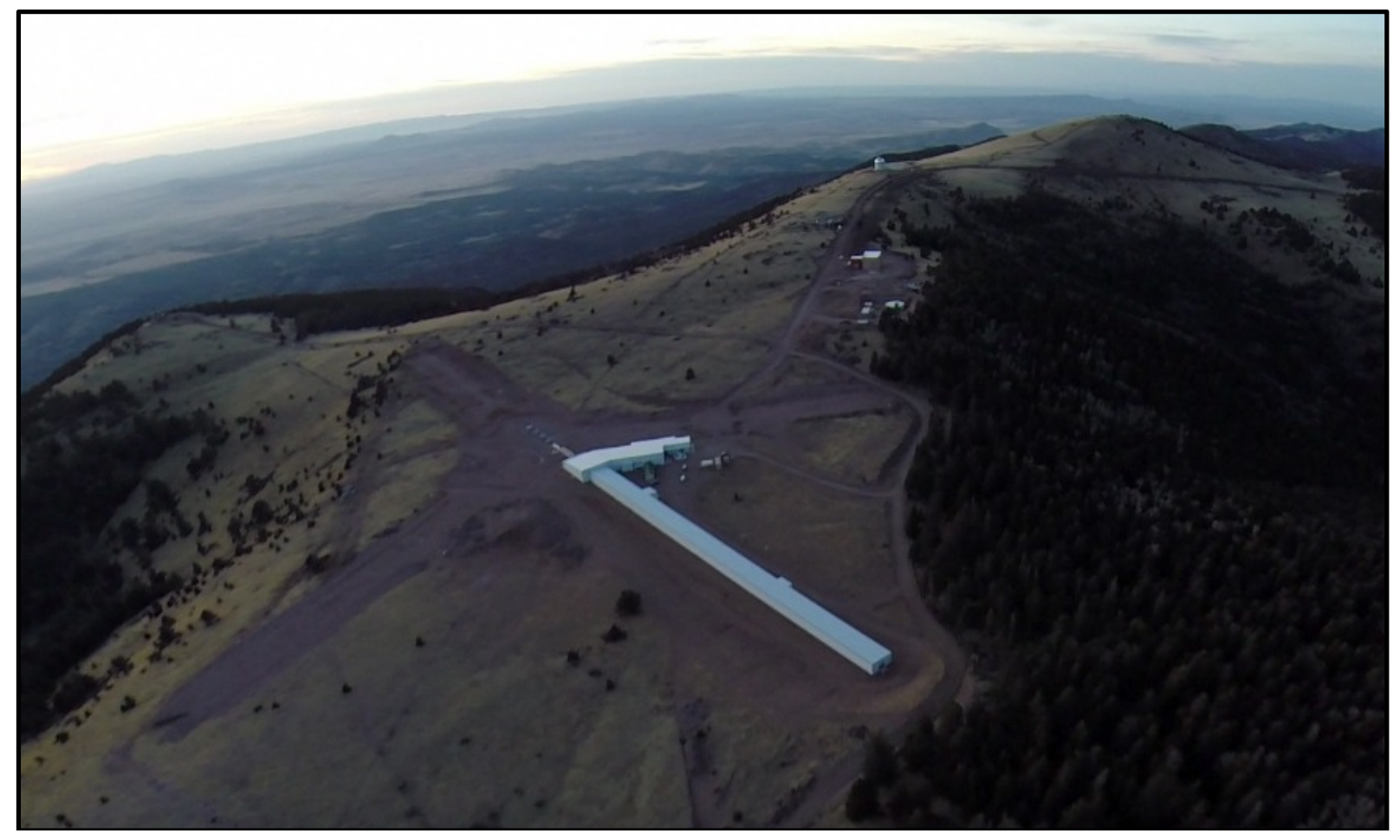

Figure 1: Overview of the Magdalena Ridge at 10,500 feet looking northwest. In the foreground is the beam combining facility and the completed array arms for the MRO interferometer. Far in the background is the $2.4 \mathrm{~m}$ fast-tracking telescope. The location of the NRAO EVLA is on the plains below in the upper left of this image. (Picture courtesy of Bob Martin, Albuquerque, NM).

\section{PROGRESS ON INDIVIDUAL SUBSYSTEMS}

The MRO interferometer (MROI) design and build activities are managed under a Work Breakdown Structure (WBS) which includes 18 separate major packages. Each of these packages includes a set of design requirements, a group of design memos and implementation decisions, and a series of formal reviews, frequently using external experts for validation of the team's conclusions, and finally resulting in a system design for each WBS item. As part of the verification of a package, risk-reduction experiments or prototype hardware may be built before a final design is agreed upon. Each full system design also requires a comprehensive assembly, integration and verification plan (AIV) so that necessary equipment and personnel resources can be predicted well in advance of deploying equipment on the mountain. Software control, which is integrated into all of the MROI's subsystems, is managed centrally and had been designed to allow for upgrades and improvements to be deployed easily throughout the life of the facility (see Farris et al., 2010). A comprehensive software system simulation framework is being developed to support testing of individual systems as they are delivered to the observatory. The current status of these 18 major workpackages is that the majority have passed their final design review (FDR), and design has been completed and is being tested in the laboratory. Below, we provide updates on several of the major WBS items, especially those where there has been significant progress over the past two years.

\subsection{Unit Telescopes and Optics}

Each MROI unit telescope (UT) consists of a $1.4 \mathrm{~m}$ diameter telescope on an altitude-altitude mount utilizing only a primary, secondary and tertiary mirror to inject the starlight into the fast tip-tilt system (mounted on a Nasmyth table and controlling the secondary mirror) and then into the beam transport system (see below). The telescopes are being built by AMOS in Liege, Belgium. Six full sets of optics have been procured for the program, with all secondary and tertiary mirrors complete, and three primaries partially fabricated at OST in Albuquerque, NM. OST folded during the economic 
downturn and we will need to find a new manufacturer to complete the primaries for the MROI. When polished and coated, the telescope optics (three mirrors together) will produce beams with a total RMS wavefront error of $63 \mathrm{~nm}$, which includes a contribution from all alignment errors. The reader can refer to Pirnay et al. 2008 and Pirnay et al. 2010 for details of the design and assembly, and integration and testing of the telescope mounts.

The first MROI telescope has completed its factory acceptance testing (FAT) and is presently located in a warehouse on the NMT campus awaiting transportation to the Ridge and site acceptance testing (SAT) later in 2014. This testing will be undertaken at the MRO Visitors Center and Maintenance Facility (VCMF) (see more below). Procurement and construction of the large components for the second and third telescopes has begin, as has initial integration of the largest parts, but additional funding is needed to bring these to a completed state (Figure 2).

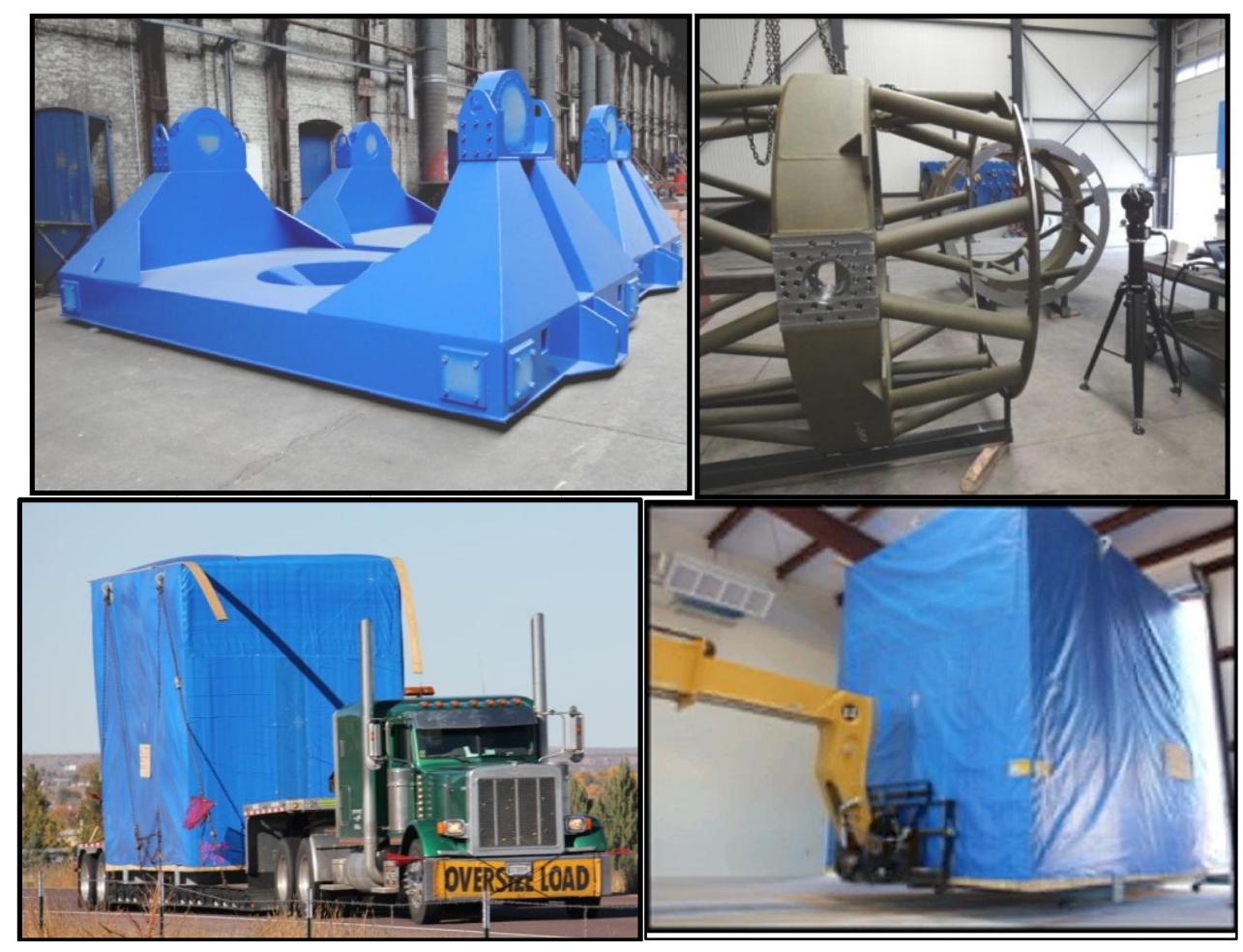

Figure 2: Telescope mounts and UT \#1 for the MROI: At top left are the bases for UTS \#2 and \#3 at the AMOS factory; at top right their corresponding two top ends. At bottom left is shown the first telescope arriving at NMT, and bottom right being received at a storage facility. The UT \#1 is now awaiting shipment to the Ridge for SAT this fall. (Top pictures are courtesy of AMOS.)

\subsection{Unit Telescope Enclosures}

The unit telescope enclosures (UTEs) for the MROI have an unusual and novel design which serves three different purposes simultaneously: 1) they protect the telescopes and their instrumentation from environmental influences, 2) they allow for the field-of-view and field-of-regard requirements for the array, especially during observations in a closepacked configuration when telescopes are separated by $7.8 \mathrm{~m}$ on centers, and 3) they allow for protected transportation of the telescopes to new configurations on the ridge to match different scientific goals (Figure 3). The UTEs have been designed by EIE in Venice, Italy (see Busatta, A. et al. 2010 and Payne, Marchiori \& Busatta, 2010). In 2011 the fabrication vendor, Berengo-Galbiati in Lecco, Italy, was competitively selected to undertake the build and deployment 
of the UTEs. We anticipate approximately 12 months of activity to produce the first UTE, which will commence as soon as funds become available.

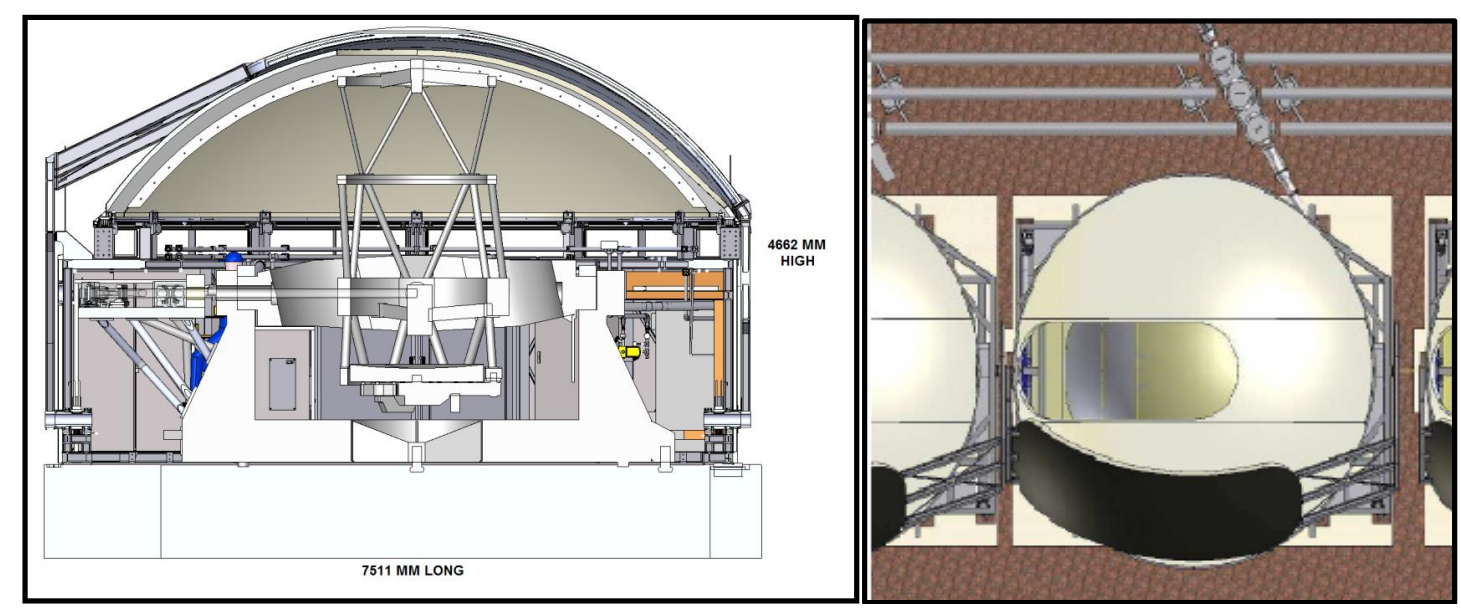

Figure 3: Two views of the MROI UT enclosures. On the left, note the efficient use of interior space, the "squat" design of the enclosure to meet the field-of-view requirements for the array for six-hour tracks, and the separated foundations for the telescope and enclosure mounts. On the right, the UT enclosures in their close-packed configuration have approximately $25 \mathrm{~cm}$ separation between adjacent telescope foundations. (These design drawings are courtesy of EIE.)

\subsection{Visitor's Center and Maintenance Facility (VCMF)}

A competitively selected proposal from the New Mexico Department of Transportation for a Visitor's Center and Maintenance Facility at Magdalena Ridge was awarded in 2011. The VCMF will include the following: visitor's area for viewing the mountain and maintenance facility bay, local information packets and displays, a gift shop, public meeting area for tours, restroom facilities, and a co-located maintenance facility for observatory equipment adjacent to the building (Figure 4). In particular, the maintenance part of the facility will house a servicing location for large earthmoving and scientific equipment from the observatory, including a telescope mounting pad, associated telescope power/cooling infrastructure, and large roll-up door for testing/debugging the MROI unit telescopes with starlight. The design was completed by URS Corporation and their sub-contractor SMPC Architects in Albuquerque, NM. The facility infrastructure is complete and we are awaiting a power transformer in order to take occupancy of the building.

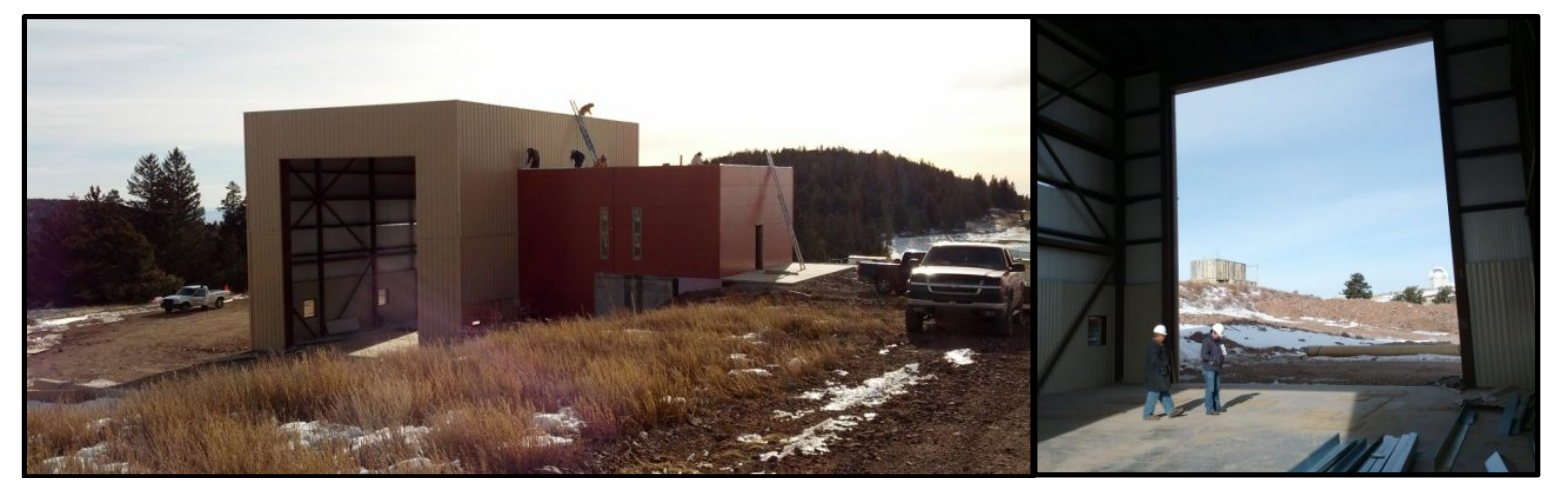




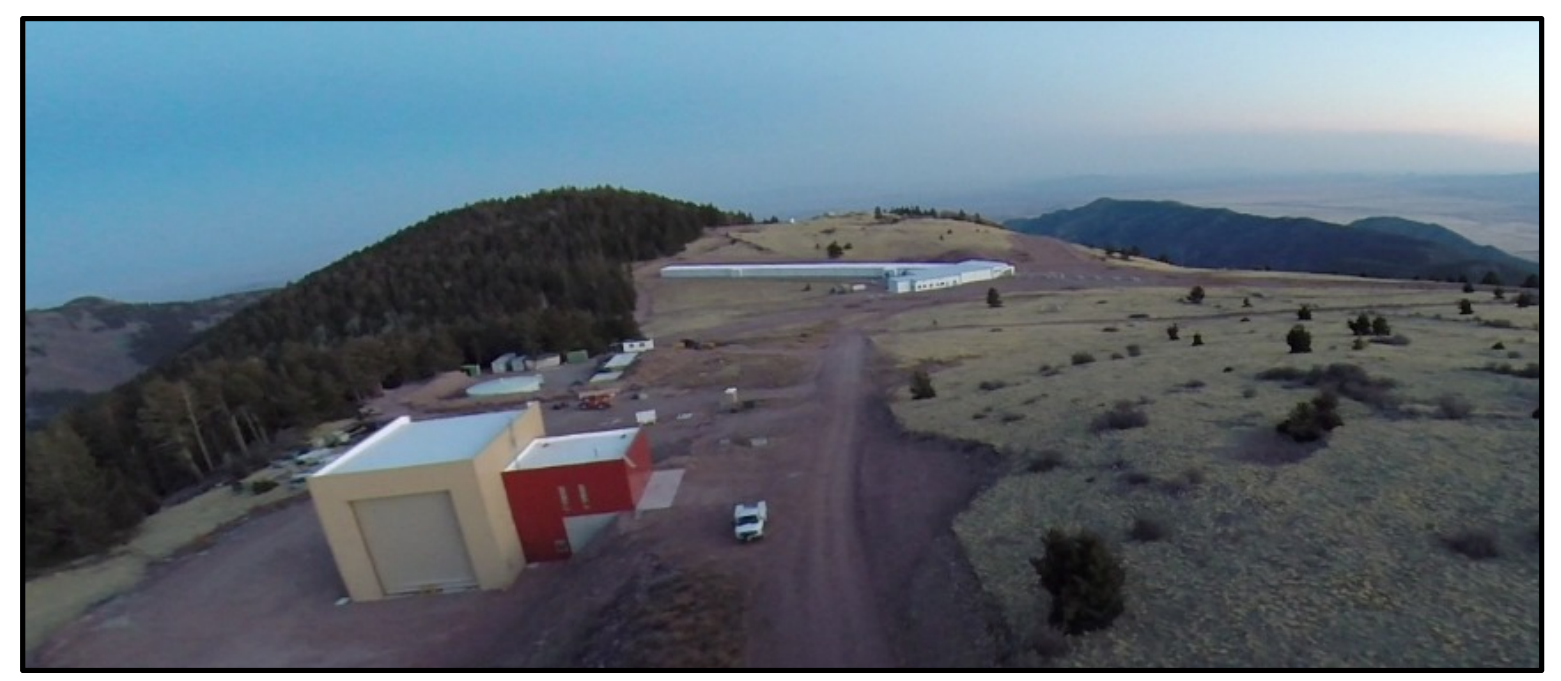

Figure 4: New NM State Dept. of Transportation funded Visitor's Center and Maintenance Facility (VCMF) at MROI. On the top left is shown the maintenance facility (beige) adjacent to the public visitor's center (red) looking toward the south. On the top right is a view from the north door inside the maintenance facility where the unit telescopes will be tested and serviced. On the bottom is a sunset view of the VCMF and MROI in the background as seen from the air (Picture courtesy of Bob Martin, Albuquerque, NM).

\subsection{Array Infrastructure and Beam Relay Systems}

When fully completed, the MROI array infrastructure will include 28 separate telescope mounting platforms for the full ten-telescope complement of the MROI, over 2 kilometers of vacuum transport pipe for the stellar beams, and close to 30 custom vacuum beam relay containers. These will be mounted on specialized platforms with stringent subsidence and vibrational stability requirements, and each will contain a beam turning mirror and its associated components required for the automated alignment system (see below). Each telescope foundation location will contain infrastructure for separate electrical, Ethernet and glycol cooling connections. Importantly, at each UT location, the UTs and UTEs will have physically decoupled kinematic mounts and foundations so that any wind-buffeting of the enclosures will not be coupled directly to the telescopes and degrade the interferometric observations.

In situ-testing and analysis of prototype components for the vacuum beam relay system demonstrated that these would not be able to meet the very stringent thermal and vibrational stability requirements needed to support the array-wide error budget. As a result, the beam relay system, which includes relay pipe supports, vacuum anchors, cross-over cans and their supports, and the mounts for mirrors 4 and 5 in the MROI beam train (the mirrors which immediately follow the telescope optics) have all undergone a complete redesign based on in-situ and lab testing of components (see Santoro et al. 2012, these proceedings). Several of these new prototypes are now installed at the Ridge and in-situ characterization for long-term beam stability is underway (Figure 5). 


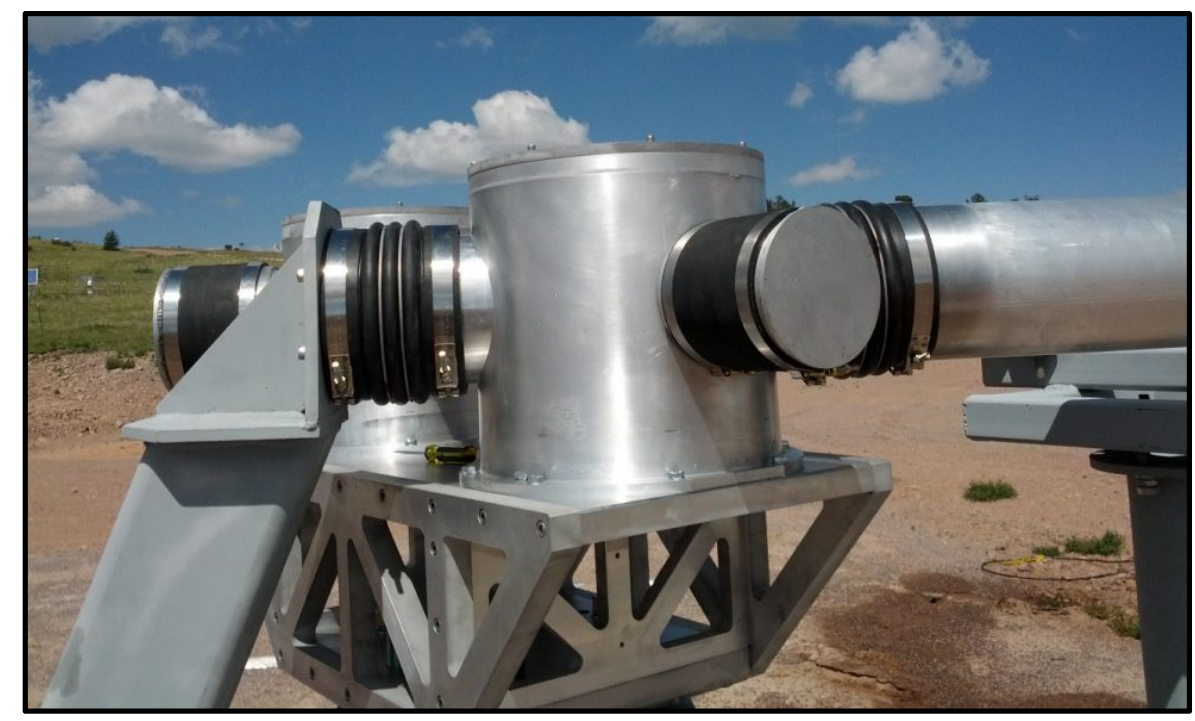

Figure 5: Telescope beam relay can with mirrors installed. This can and its associated optics are under long-term stability testing outside the beam combining facility at MROI.

Design of the array infrastructure components has been a collaborative project between the observatory and M3 in Tucson, AZ, who designed the MROI beam-combining facilities (see Creech-Eakman, et al. 2008 for details of the building design). To date, a total of seven of the inner central telescope pads have been installed at MROI, and we anticipate installing required power, cooling and optical fiber infrastructure as funds become available.

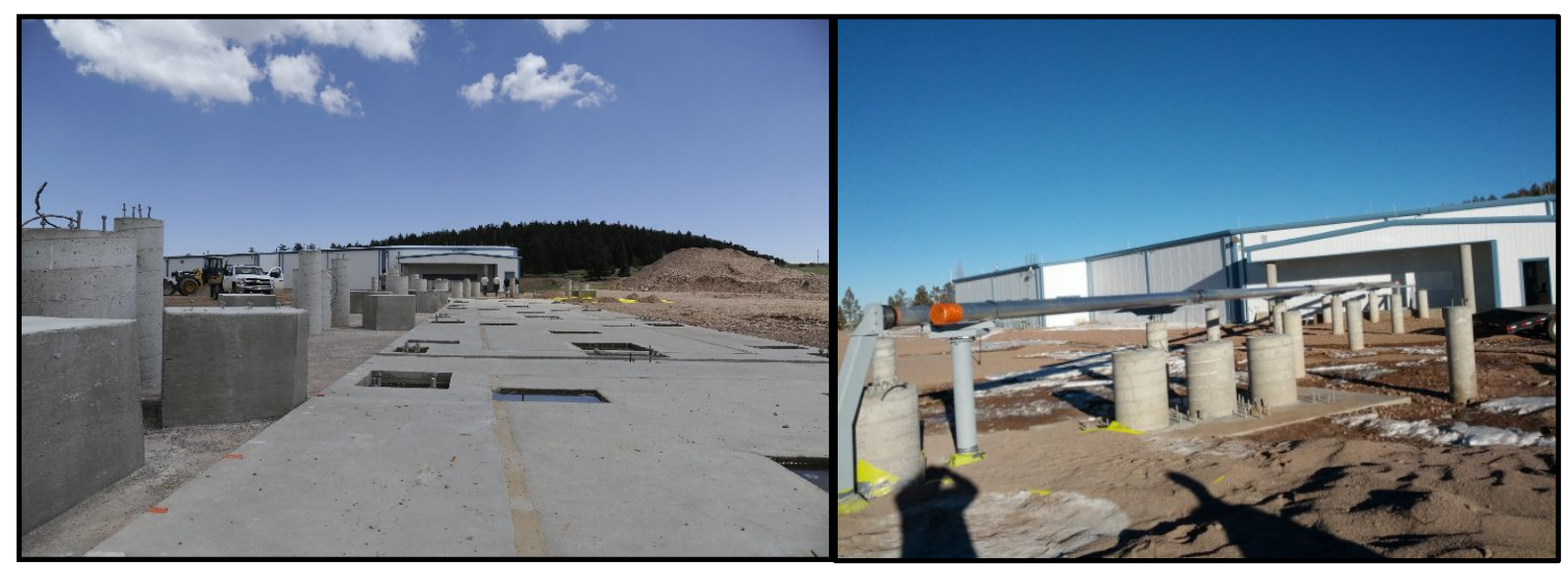

Figure 6: A view along the west array arm of the interferometer looking toward the beam combining facilities. At left are four of the telescope and enclosure foundations are shown together with the beam relay and vacuum can piers running along the left-hand side of the photo. At right, the foundations have been covered to protect them from heavy equipment, and installation of vacuum pipes has begun. 


\subsection{Fast Tip-Tilt System and Acquisition Systems}

The MROI combined fast tip-tilt/narrow-field acquisition systems are located on the Nasmyth platform of the unit telescopes. The role of the systems is to perform initial acquisition of an observational target, and subsequently to maintain the telescope pointing and remove low-order atmospheric tip-tilt perturbations by actuating the telescope's secondary mirror throughout the scientific observation. All sensing for this part of the MROI system will occur at optical wavelengths $(400-1000 \mathrm{~nm})$ so that near-infrared light is preserved for fringe-tracking and initial science. Future MROI science observations at optical wavelengths will likely involve the bluest light of this band (up to $\sim 600 \mathrm{~nm}$ ) being used for tip-tilt sensing.

The fast tip-tilt and narrow-field acquisition systems have been designed and prototyped by the University of Cambridge and are undergoing final lab testing at the time of this meeting. The details and status of the system are discussed in Young et al. (2014) these proceedings. We expect to deploy this system on the Ridge later in 2014 or early 2015, after SAT for the first telescope has been completed.

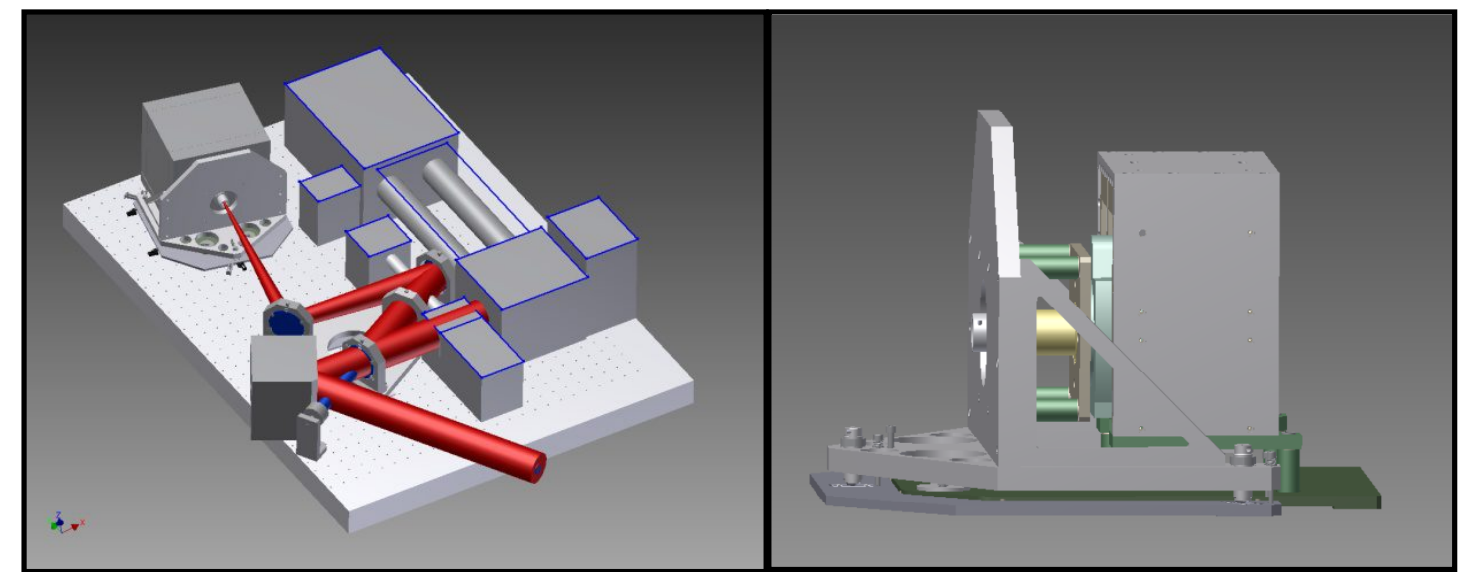

Figure 7: MROI's fast tip-tilt system. On the left is an isoplanatic view of the preliminary design for the fast tip-tilt system on the Nasmyth table of the telescope, and on the right is a side view of the camera mount. See Young et al. 2014 (these proceedings) for details.

\subsection{Delay Lines}

The delay lines and associated carts, or trolleys, are a completely innovative system in the field of optical/infrared interferometry and are being designed and fabricated by the University of Cambridge (see Fisher et al. 2010 for complete details). Risk reduction experiments and tests of a prototype trolley were initially undertaken in Cambridge, resulting in the present trolley final design. This design is the only one in existence which allows a continuous delay stroke of up to approximately 400 meters in vacuum. The MROI delay lines feature innovations in three major areas: 1) the trolleys have compliant wheels and actuated secondaries (in a cat's eye design) such that they can run directly on standard 16 inch diameter aluminum pipe, with closed-loop correction of beam shear due to pipe irregularities, 2) the optomechanical and control system design surpasses all requirements for optical path length jitter, including when passing over pipe joints in the system, and 3) the use of inductive pick-up along the bottom of the vacuum pipe and wireless communication with the on-board computer avoids the need to drag cables behind the trolley.

The delay lines passed FAT at Cambridge and SAT at Magdalena Ridge began in late 2013. The trolleys performed to specifications using the metrology system in the inner Beam Combining Area (BCA) for reference, but issues with the computer communication within the building and to the trolleys themselves delayed completion of SAT. We anticipate completing SAT on the first DL cart later this year and, with the completion of the second trolley at Cambridge, beginning SAT on the second trolley in early 2015. 


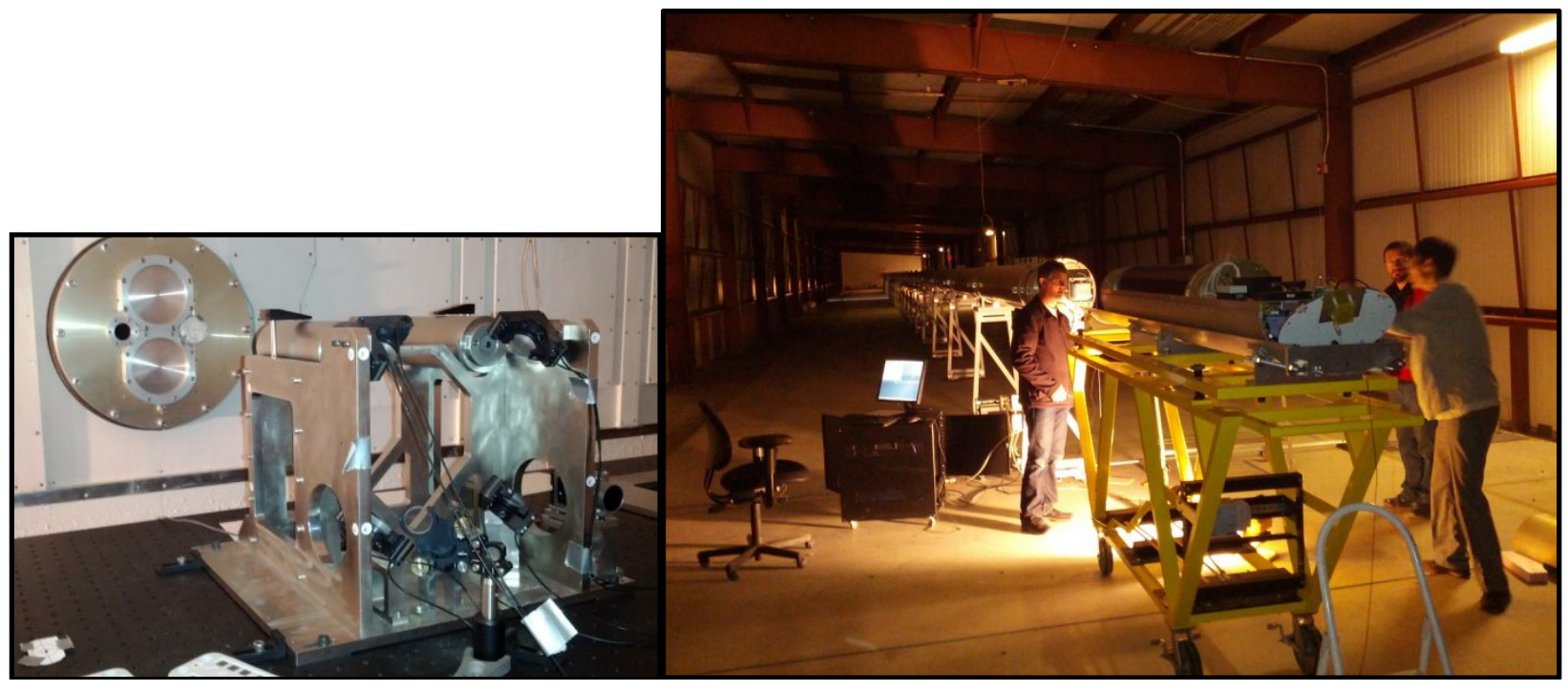

Figure 8: Successful installation of delay line pipe and trolley at MROI. On the left is shown the metrology launch table within the inner beam combining area, while on the right is an image from SAT work during the installation of trolley \#1 into the $100 \mathrm{~m}$ delay line pipe.

\subsection{Automated Alignment System}

The MROI alignment system will be a fully computer automated optical alignment system which will be used to feed and align white-light and laser light simultaneously through the entire MROI beam train (fringe tracker and science instruments, delay lines, and beam relay system out to the unit telescopes). The use of the system will greatly improve the efficiency of the initial alignment of the interferometer, and will be key to rapidly re-locating the starlight beams and getting back on-sky if an alignment fault occurs during the night (see Shtromberg et al. 2010 and Shtromberg, 2013). As part of the closed-loop fringe experiment (CLFE) (see below) a fully operational prototype system was deployed on the fringe-tracker table in the lab at NMT. Characterization and testing were completed as part of a M.S. degree (Shtromberg, 2013) and recommendations for improvements to be undertaken for deployment on the Ridge were made. These recommendations included in particular abandoning a fiber-launch design in favor of a bulk-optics design for the light launching system due to extreme losses in the single-mode fiber combiner. Discussion of the impacts of this issue is included in a more complete discussion of the ICoNN fringe-tracker testing (McCracken et al., 2014 these proceedings).

Since completion of the M.S. degree by Shtromberg, further lab testing of the algorithms for automating the MROI alignment have revealed mismatches in the theoretical model of the MROI mirror motions, and actual motions, in part due to hysteresis in the piezo mounts. A simplified optical system and updated alignment algorithm were successfully tested in the lab, resulting in the deployment of a modified system in the NMT laboratory as part of ongoing testing of the ICoNN fringe tracker. This will allow us to test complete automation of mirror motions on the table over the coming year. 


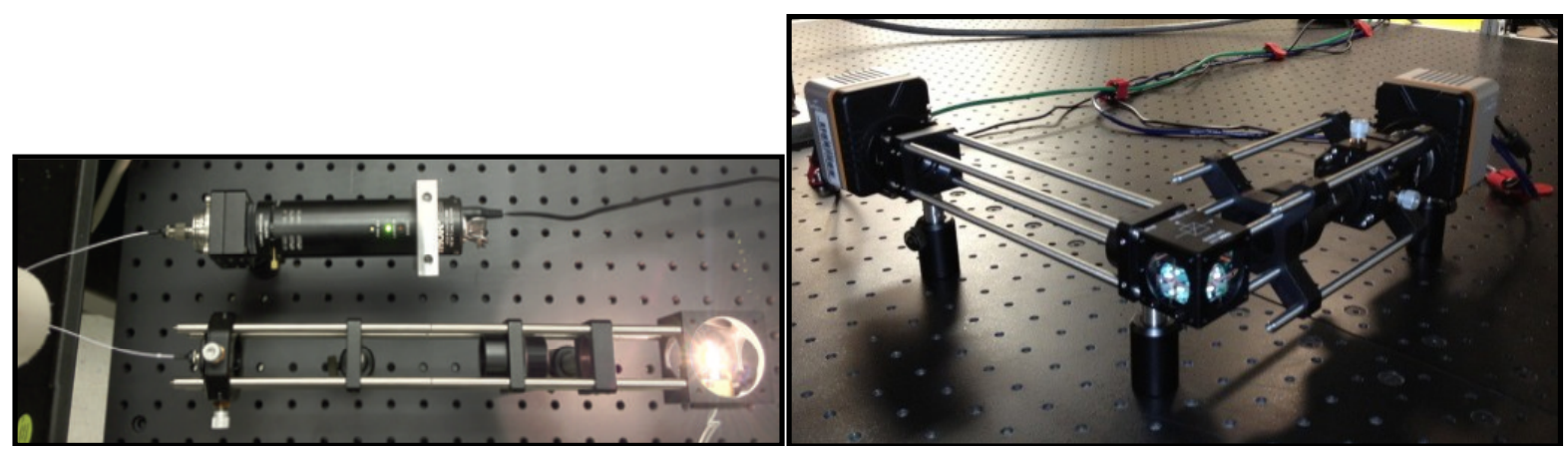

Figure 9: Components of the MROI automated alignment system. At left are the fiber injectors for the white-light and near-infrared laser alignment beams that are part of the "magical optical box" and at right are the cameras for the sensing the tilt and shear in the ICoNN system.

\subsection{Fringe-Tracker System}

The MROI fringe-tracker ICoNN (Infrared Coherencing Nearest Neighbor tracker) is a nearest neighbor style pairwise beam combiner that can mix and sense the full complement of 10 telescope beams of the MROI. The design enables ICoNN to detect and track the interferometer fringe phase at either the $\mathrm{H}_{\text {or }} \mathrm{K}_{\mathrm{s}}$ infrared bandpasses for any distribution of neighboring telescopes. It is a dedicated fringe tracker that does not share light with the separate science beam combiner(s) operating at other infrared or optical. The ultra-stable MROI design is completely non-actuated save for its modulating mirrors, and has demonstrated excellent opto-mechanical stability even when tested in a university laboratory environment with no temperature or vibration control. Previous proceedings (Jurgenson et al. 2008 and McCracken et al. 2012, Santoro et al. 2012) have detailed the design and plans for laboratory testing of the ICoNN system. Use of ICoNN will enable the MROI science case, which has been recently expanded to include studies of geosynchronous satellites (Young, Haniff \& Buscher, 2013), especially as implemented in the 10 telescope MROI deployment.

Since the last SPIE meeting we have closed-the-loop in the lab using ICoNN to track fringes with a mock delay line plus "atmospheric disturbance" and real-time control, demonstrating that ICoNN can routinely track fringes to less than a 5\% coherence loss. We have also tested the open-loop modulation scheme in detail for the system (see McCracken et al. 2013 for details of the modulation, and Jurgenson et al. 2013 and McCracken et al. 2014 these proceedings for details of the ICoNN assembly, integration, testing and present status). McCracken completed his dissertation work on the CLFE experiment with the custom modulation scheme for ICoNN (McCracken, 2014) and has taken a postdoctoral appointment at Yale University.

We have recently received all internal cold optics (see Figure 10) and their custom mounts for ICoNN and are planning to install and test them in the NMT laboratory over the next year. To make full use of these new optics, we also have developed a new five-line readout mode for the existing PICNIC detector which has a $500 \mathrm{~Hz}$ frame rate before including the fringe tracking algorithms. Custom periscope optics fabrications are delayed and will be built as funds become available. In the long-term, we intend to install an infrared eAPD, likely a SELEX SAPHIRA detector (see Finger et al. 2014 and Buscher et al. 2014), into the ICoNN dewars, allowing us to achieve $14^{\text {th }}$ magnitude at $\mathrm{H}$ band, as designed to reach our AGN science targets. 


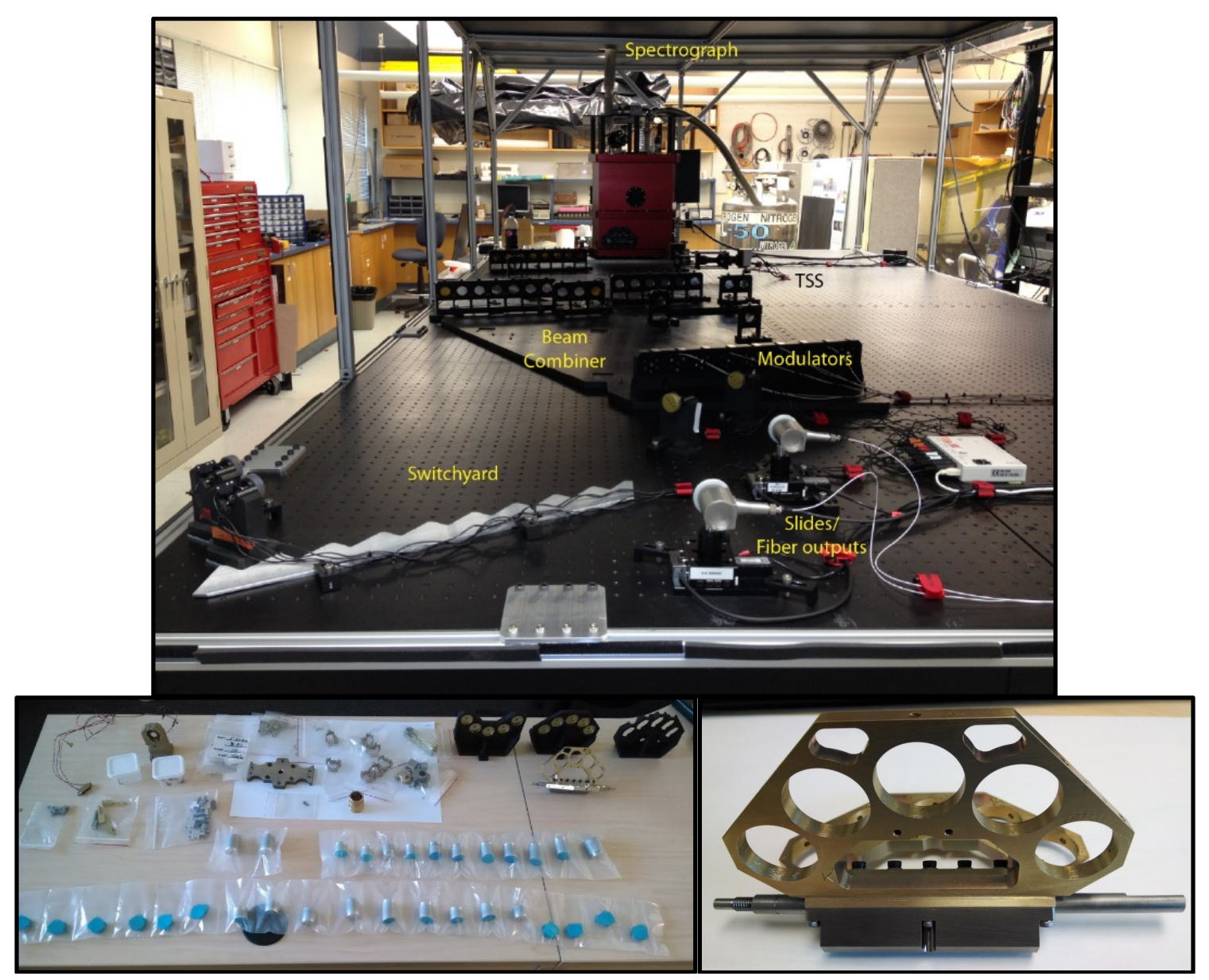

Figure 10: The MROI fringe tracker, ICoNN, under test in the lab. The upper panel shows the beam combiner, spectrograph, modulators and switchyard being fiber-fed by light from the automated alignment system in the foreground of the picture. The lower left hand panel shows newly-arrived cold optics for five beam ports being prepped for installation, and on the lower right is a close up of the filter "flipper mechanism", used to swiftly change filters and save space on the cryo-surface.

\section{FUNDING AND THE SCHEDULE FOR FIRST LIGHT}

The schedule for first-light with the MROI is strongly tied to the observatory funding profile. At present we are pursuing multiple avenues of funding including further Federal, State, institutional and philanthropic sources and are looking for partner institutions to join us to help complete our vision of the MROI. A near-term aggressive funding profile, with the goal of attaining fringes in the next two years, will allow us to reach the following milestones for the observatory by the next SPIE meeting in Edinburgh:

- 2014: Completing SAT on the first telescope and first delay line trolley. Initiating contracts to complete the first UT enclosure and begin subsequent enclosures. Initiating contracts to complete telescope primary figures to specifications. Initiating contract to complete telescopes 2 (and 3 if funding permits).

- 2015: Completing necessary infrastructure (power, cooling, fiber, beam relay, etc.) on the Ridge outdoors to execute first-fringes in 2016. Performing SAT on the FTT system at the VCMF. Adding internal infrastructure 
(optical tables, DL and beam transport pipes and optics) to Ridge facilities indoors to support attempt for first fringes in 2016. Completing periscope optics and installing ICoNN in the inner BCA.

- 2016: Receiving and installing telescope and enclosures 1 and 2 at MROI. Attaining first fringe measurements using two MROI telescopes.

The business plan and operational model for the MROI facility have been completed and ideas associated with operating observatories in the current economic climate are discussed in Payne and Veillet (2014). We are presently favoring a consortium-style model for operations of the MROI facility such that individual investigators, university partners, and private groups can all attain access to MROI's capabilities in an al a carte type menu. We anticipate initial operations beginning coincident with the deployment of the scientific beam combiner instrument and 4-telescope operability at MROI. Interested parties should contract the observatory director, Ifan Payne, directly to initiate discussions.

\section{ACKNOWLEDGEMENTS}

The Magdalena Ridge Observatory Interferometer is funded by the U.S. Department of Transportation, the State of New Mexico, and New Mexico Tech with previous funding from the Navy Research Laboratory (NRL, agreement no. N00173-01-2-C902). MROI is hosted by New Mexico Tech at Socorro, NM, USA, in collaboration with the University of Cambridge (UK).

\section{REFERENCES}

Buscher, D. F., Creech-Eakman, M., Farris, A., Haniff, C. and Young, J., "The Conceptual Design of the Magdalena Ridge Observatory Interferometer," Journal of Astronomical Instrumentation, 2, 2, 1340001, (2013).

Buscher, D. F. et al. "Optimisation of infrared eAPD detector readout for interferometry", Proceedings of SPIE, 9146, these proceedings, (2014).

Bussata, A. et al. "Innovative enclosure dome/observing aperture system design for the MROI Array Telescopes", Proceedings of SPIE, 7739, 59B, (2010).

Creech-Eakman, M. J. et al. "Magdalena Ridge Observatory Interferometer: progress toward first light", Proceedings of SPIE, 7013, 26C, (2008).

Creech-Eakman, M. J. et al. "Magdalena Ridge Observatory Interferometer: a status update”, Proceedings of SPIE, 8445, OP, (2012).

Farris, A. et al. "Software architecture of the Magdalena Ridge Observatory Interferometer" Proceedings of SPIE, 7740, 24F, (2010).

Finger, G., et al. "SAPHIRA detector for infrared wavefront sensing", Proceedings of SPIE, 9148, these proceedings (2014).

Fisher, M. et al. "Design of the MROI delay line optical path compensator", Proceedings of SPIE, 7734, 135 (2010).

Jurgenson, C. et al. "Fringe Tracking at the MROI", Proceedings of SPIE, 7013, 37J, (2008).

Jurgenson, C. et al., "ICoNN - The Infrared Coherencing Nearest Neighbor Tracker", Journal of Astronomical Instrumentation, 2, 2, 1340006, (2013). 
McCracken, T. et al. "The MROI Fringe Tracker: Closing the loop on ICoNN”, Proceedings of SPIE, 8445, (2012).

McCracken, T. et al. "Open-loop phase shifting for fast acquisition of interferograms in low light levels", Applied Optics, 52, 20, p 4922-4932, (2013).

McCracken, T. “The ICoNN Fringe Tracker”, PhD Dissertation, New Mexico Institute of Mining and Technology, (2014).

Payne, I., Marchiori, G. and Busatta, A. "Innovative enclosure design for the MROI array telescopes", Proceedings of SPIE, 7739, 49P, (2010).

Payne, I. and Veillet, C. “Operating observatories: the need for a new paradigm”, Proceedings of SPIE, 9149, these proceedings, (2014).

Pirnay, O. et al., "Design of the unit telescopes of the MROI", Proceedings of SPIE, 7013, 19P, (2008).

Pirnay, O. et al. "Magdalena Ridge Interferometer: assembly, integration and testing of the unit telescopes", Proceedings of SPIE, 7734, 31P, (2010).

Santoro, F. et al. "Final Mechanical and Opto-Mechanical Design of the Magdalena Ridge Observatory Interferometer", 2012, Proceedings of SPIE, 8445, 2KS.

Shtromberg, A. et al. "Magdalena Ridge Observatory Interferometer automated alignment system", Proceedings of SPIE, 7734, 32S, (2010).

Shtromberg, A. V. "Magdalena Ridge Observatory Interferometer Automated Alignment System", M.S. Thesis, New Mexico Institute of Mining and Technology, (2013).

Young, J. S. et al. "Performance of the MROI fast tip-tilt correction system", Proceedings of SPIE, 9146, these proceedings, (2014).

Young, J. S., Haniff, C. and Buscher, D., "Interferometric Imaging of Geo-synchronous Satellites with Ground-based Telescopes", IEEE, Proceedings of the Aerospace Conference, March 2-9, Big Sky, MT, USA, ISBN: 978-1-4673-18129, id.93, (2013). 